\title{
Is Social Distancing the Unvaccinated Justifiable?
}

\author{
Paul T Francis* \\ Department of Community Medicine, Amrita Institute of Medical Sciences, India
}

*Corresponding author: Dr Paul T Francis, MD, Associate Professor, Department of Community Medicine, Amrita Institute of Medical Sciences, Cochin, 682041 , India

Submission: 惯 September 22, 2017; Published:

\section{Introduction}

Social distancing the unvaccinated has become an issue recently with the 'Disneyland outbreak' of measles in United States [1]. Ever since the Wakefield article (later retracted) linking MMR vaccine to autism, the issue of vaccination and so called vaccine-refusal has on and off been in the news. The American Academy of Pediatrics has even issued guidelines regarding denial of service to unvaccinated children [2,3]. A New York court has ruled in January 2015 that "the state can continue requiring public school students to be vaccinated or excluded during outbreaks of vaccine-preventable diseases" [4].

The article discusses the presumptions and rationale for excluding the unvaccinated children from school during outbreaks. The author will not refer to the legality of it, because it is not his area of expertise. The author will also not discuss the broader issue of vaccine safety and effectiveness, because it goes beyond the scope of this analysis. These exemptions had to be explicitly stated, because the decision on social distancing is largely based on these two important foundations.

Social distancing is based on the 'fact' that the unvaccinated is at risk of getting the disease and more importantly, is responsible for spreading the disease to others [5]. The presumption is, being unvaccinated did not develop immunity. Subclinical infections occurring naturally can probably give better immunity than artificially induced vaccine immunity. Limits of germ theory of disease should caution us against accepting vaccination as the only (effective) method to prevent infectious diseases.

No vaccine has $100 \%$ efficacy and can guarantee immunity for an assured period of time [6]. So there is a proportion of children who are vaccinated but not immune. The vaccinated also can get infected and excrete/shed the organism in question. Those who are vaccinated against Influenza are sometimes required to take antivirals during an outbreak [7]. So can we accuse only the unvaccinated for allegedly spreading the disease, when the vaccinated also may do so? Despite great advances in immunology our knowledge about immune system is very limited.

It is in this scenario an outbreak is occurring and there is a demand for excluding the unvaccinated from school. Since the unvaccinated is aware of the risk of getting the disease, they (or parents) will not blame anyone for getting the disease. So in most circumstances the parents of unvaccinated, will not demand for any special protection. And the risk of infection is not specific to attending school.

Since the vaccinated children (most are) are protected against the disease, they should not fear interacting with the unvaccinated. So for whose protection is the social distancing enforced? It appears that the only persons who could possibly benefit from social distancing are those who failed to develop immunity after vaccination, which is not a justification enough for excluding healthy children from school. This is a blind and senseless retribution because we are subconsciously demonizing many relatively mild and treatable infectious diseases just because there is a vaccine. Are we penalizing them after accusing them as 'free riders' for no fault of theirs?

The extreme punitive action of denying medical care to the unvaccinated, is done on the surmise that the child (or adult) is refusing a protective measure against disease and hence should pay for it. Unavailability of a vaccine currently does not make any disease vaccine-unpreventable. Using the more appropriate term vaccine-available disease, will create a paradigm shift in our attitude towards the so called vaccine-deniers. There are many known risk factors for many diseases, if removed can prevent the diseases. We do not refuse medical care to an obese smoker with angina-pectoris telling him/her to comeback after reducing weight and quit smoking. We provide all sorts of care to the practically incurable disease in a obese smoker, which are prohibitively expensive, but righteously deny antipyretics and antibiotics to unvaccinated, which cost a minuscule.

Should we not 'punish' those who smoke, overindulge in alcohol, overeat etc. leading to severe, chronic and fatal diseases? Will we socially distance a smoker from school/college lest he/she be a bad influence on others? Improvement in living conditions, nutrition and environment, advances in medicine, microbiology and epidemiology have made most communicable diseases negligible in incidence and clinically manageable, in the developed countries. There is definitely no need to be paranoid about relatively harmless communicable diseases and embark on inquisition against those 
who chose not to vaccinate. The morbidity and mortality due to these infectious diseases is relatively insignificant compared to that of non-communicable diseases. It is strange and unfortunate that when it comes to the issue of vaccination Public Health officials and some clinicians tend to lose their perspective and miss the elephant in the room, which is non-communicable diseases. If we are angry at 'vaccine-deniers' shouldn't we be more so at 'exercise-deniers'?

\section{References}

1. Centers for Disease Control and Prevention (2015) Measles cases and outbreaks.

2. American Academy of Pediatrics (2013) Reaffirmation: responding to parents who refuse immunization for their children. Pediatrics 131(5): e1696-e16.

3. Diekema DS (2005) Responding to Parental Refusals of Immunization of Children. Pediatrics 115(5):1428-1431.

4. Phillips v (2015) City of New York, No. 14-2156 (2d Cir).

5. Yang YT, Silverman RT (2015) Social Distancing and the Unvaccinated. N Engl j med 372(16): 1481-1483.

6. Poland GA, Jacobson RM (2012) The Re-Emergence of Measles in Developed Countries: Time to Develop the Next-Generation Measles Vaccines? Vaccine 30(2): 103-104.

7. Gardam M, Lemeiux C (2013) Mandatory influenza vaccination? First we need a better vaccine CMAJ 185(8): 639-640. 